\title{
Results from the forward G0 experiment
}

\section{Strange quark contribution to the nucleon structure}

\author{
J.-S. Real ${ }^{\mathrm{a}}$ \\ For the G0 Collaboration ${ }^{\mathrm{b}}$ \\ LPSC, IN2P3-UJF, 53 Avenue des Martyrs, 38026 Grenoble Cedex, France
}

Received: 31 August 2006

Published online: 28 June 2007 - (C) Società Italiana di Fisica / Springer-Verlag 2007

\begin{abstract}
The G0 experiment is dedicated to the determination of the strange quark contribution to the electric and magnetic nucleon form factors for a large range of momentum transfers between 0.1 to $1(\mathrm{GeV} / c)^{2}$. This information is provided by the asymmetries of cross-sections measured with longitudinally polarized electrons in elastic electron-proton scattering and quasi-elastic electron-deuteron scattering. A set of measurements at two different $Q^{2}$ will allow the complete separation of the electric and magnetic weak, as well as axial nucleon form factors. This report summarizes the physics case, gives details about the dedicated set-up used, and shows the results of the combination of the strange quark contribution in the electric and magnetic form factors of the protons. The experiment was performed at the Jefferson Laboratory, during years 2003 and 2004, and will be completed after backward-angle measurements in 2006, 2007.
\end{abstract}

PACS. 13.60.Fz Elastic and Compton scattering - 13.40.Gp Electromagnetic Form Factors - 14.20.Dh Protons and neutrons

\section{Introduction}

In quantum chromodynamics, the nucleon is viewed as composed of three-valence $u$ and $d$ quarks and a sea of gluons and quark-antiquark pairs $(u \bar{u}, d \bar{d}, s \bar{s}, \ldots)$. Because the strange quarks contribute only to the sea, their contribution to the nucleon properties is one of the open questions, which are investigated in a joint experimental and theoretical study.

Several indications already exist on the strange quark contribution to nucleon properties, as to the momentum with deep inelastic neutrino scattering, to the spin with deep inelastic electron scattering and to the mass from $\pi$-nucleon scattering. All these experiments provide contributions ranging from 0 to $30 \%$ with large theoretical and experimental uncertainties.

The parity-violating (PV) electro-weak interaction in electron-nucleon elastic scattering offer a new opportunity to study the contribution of strange quarks to the electroweak structure of the nucleon through the nucleon form factors, and in particular possible difference in the $s$ and $\bar{s}$ spatial densities.

\footnotetext{
a e-mail: real@in2p3.fr

b Jefferson Lab Experiments E-05-108/06-108 and E-04-101. A list of collaborators and information about the experiment can be found at http://www.npl.uiuc.edu/exp/G0/.
}

Several dedicated PV asymmetry experiments and apparatus have been developed during the last decade for these studies [1]. In this contribution we focus on the G0 experiment which provides a $Q^{2}$-dependence of the linear combination of the strange magnetic and electric form factors over a large range of momentum transfers $\left(Q^{2}\right.$ between 0.1 to $\left.1(\mathrm{GeV} / c)^{2}\right)$, and will provide a complete determination of the contribution of the strange quarks to the electric, magnetic and axial form factors.

In the following, we will present the physics motivation before describing the G0 experimental set-up. Then, we will show the results of the G0 experiment in the forwardangle configuration which provide the first determination of a linear combination of the strange contribution to the electric and magnetic nucleon form factors over a large $Q^{2}$ range.

\section{Elastic scattering and parity-violating experiment}

In electron-nucleon scattering, the electroweak interaction takes place at first order through two diagrams, one corresponding to the exchange of a virtual photon $\left(\mathcal{M}_{\gamma}\right)$ and one to the exchange of a $Z^{0}\left(\mathcal{M}_{Z}\right)$ with a 4 -momentum transferred $-Q^{2}=\nu^{2}-\boldsymbol{q}^{2}$.

The scattering amplitudes are expressed in terms of the leptonic and hadronic currents. The hadronic currents 
associated to the virtual photon and the $Z^{0}$ can be decomposed through a multipole expansion in terms of the electromagnetic proton form factors $G_{E}^{(\gamma, p)}$ and $G_{M}^{(\gamma, p)}$ and the electric $G_{E}^{(Z, p)}$, magnetic $G_{M}^{(Z, p)}$ and axial $G_{A}^{e p}$ weak form factors.

In order to provide information on the spatial distribution of the quark inside the nucleon, the form factors can be decomposed in terms of their quark contribution separately for the electromagnetic, vector and axial weak parts as

$$
\begin{aligned}
G_{E, M}^{(\gamma, N)} & =\sum_{q=u, d, s} Q_{q} G_{E, M}^{(q, N)}, \\
G_{E, M}^{(Z, N)} & =\sum_{q=u, d, s} C_{V}^{q} G_{E, M}^{(q, N)}, \\
G_{A}^{e N} & =\sum_{q=u, d, s} C_{A}^{q} G_{A}^{(q, N)} .
\end{aligned}
$$

The contribution of the three heavy flavors has been neglected due to their larger mass. Using the charge symmetry approximation under the exchange of $u$ and $d$ quarks $\left(G_{E, M}^{(u, p)}=G_{E, M}^{(d, n)}\right)$ and $s$ quark $\left(G_{E, M}^{(s, p)}=G_{E, M}^{(s, n)}\right)$, it is possible to express the electric and magnetic quark form factors as a function of electromagnetic and weak form factors of the proton and neutron.

Because the weak interaction does not conserve parity, $\mathcal{M}_{Z}$ can be accessible through the asymmetry measurement of the cross-sections associated to elastic scattering of longitudinally polarized electrons with two opposite helicities:

$$
\begin{aligned}
A_{P V} & =\frac{\left|\mathcal{M}^{+}\right|^{2}-\left|\mathcal{M}^{-}\right|^{2}}{\left|\mathcal{M}^{+}\right|^{2}+\left|\mathcal{M}^{-}\right|^{2}} \\
& \simeq \frac{\mathcal{R} e\left(\mathcal{M}_{\gamma} \mathcal{M}_{Z}^{*}\right)^{+}-\mathcal{R} e\left(\mathcal{M}_{\gamma} \mathcal{M}_{Z}^{*}\right)^{-}}{2\left|\mathcal{M}_{\gamma}\right|^{2}} .
\end{aligned}
$$

That can be rewritten

$$
A_{P V}=a \frac{\epsilon G_{E}^{(\gamma, p)} G_{E}^{(Z, p)}+\tau G_{M}^{(\gamma, p)} G_{M}^{(Z, p)}-b \epsilon^{\prime} G_{M}^{(\gamma, p)} G_{A}^{e p}}{\epsilon\left(G_{E}^{(\gamma, p)}\right)^{2}+\tau\left(G_{M}^{(\gamma, p)}\right)^{2}}
$$

with $\tau=\frac{Q^{2}}{4 M^{2}}$ and

$$
\begin{aligned}
\epsilon=1 /\left(1+2(1+\tau) \tan ^{2} \theta_{e} / 2\right), & a=G_{F} Q^{2} /(4 \sqrt{2} \pi \alpha), \\
\epsilon^{\prime}=\sqrt{\tau(1+\tau)\left(1-\epsilon^{2}\right)}, & b=1-4 \sin ^{2} \theta_{W} .
\end{aligned}
$$

Using eq. (1) to express $G_{E M}^{Z}$ in terms of $G_{E M}^{p}$ and $G_{E M}^{s}$ :

$$
A_{P V}=A_{0}+\alpha G_{E}^{s}+\beta G_{M}^{s}+\delta G_{A}^{e}(T=1),
$$

where $\alpha, \beta$ and $\delta$ contain kinematics factor and known electromagnetic form factors.

Asymmetry as small as few $10^{-6}$ can be extracted with few $\%$ of precision thanks to the cancellation of normalization systematic errors in the ratio of the cross-section measurements. This expression shows that the asymmetry is already non-zero even if the strange quarks do not contribute to the nucleon properties.
A complete separation of the three form factors $G_{E}^{s}$, $G_{M}^{s}$ and $G_{A}^{e}$ require three independent measurements. This could be performed by varying the kinematical factors $\left(\epsilon\right.$ and $\left.\epsilon^{\prime}\right)$ at a fixed $Q^{2}$. Forward and backward (scattered electron) angles measurements correspond, respectively, to small $\epsilon$ (large $\epsilon^{\prime}$ ) and large $\epsilon$ (small $\left.\epsilon^{\prime}\right)$. As a third measurement, it appears more effective [2] to measure the asymmetry in quasi-elastic scattering on deuterium, which involves the proton and the neutron.

The HAPPEX experiment [3], performed in Hall A of Jefferson Laboratory, has measured the PV asymmetry on the proton at forward angles for $Q^{2}=0.1$ and $0.48(\mathrm{GeV} / c)^{2}$, which allowed the extraction of a linear combination $G_{E}^{s}+\eta G_{M}^{s}$, and on ${ }^{4} \mathrm{He}$ at $Q^{2}=0.1$ which allowed the extraction of $G_{E}^{s}$. The PVA4 experiment [4], performed at the MAMI facility, has measured asymmetry on the proton at forward angles for $Q^{2}=0.1$ and $0.23(\mathrm{GeV} / c)^{2}$, and is sensitive to the combination $G_{E}^{s}+\eta G_{M}^{s}$. The SAMPLE experiment at Bates, has measured the asymmetries on proton and deuteron for backward angles of $Q^{2}=0.04$ and $0.1(\mathrm{GeV} / c)^{2}$ [5], which allowed the separation of the magnetic $G_{M}^{s}$ and axial $G_{A}^{e}$ form factors.

The G0 Collaboration proposes to perform the complete separation of the electric $G_{E}^{s}$, magnetic $G_{M}^{s}$ and axial $G_{A}^{e p}$ form factors for two different momentum transfers 0.23 and $0.6(\mathrm{GeV} / c)^{2}$. First an asymmetry measurement has been performed for forward electron scattering angles between 7 and $15^{\circ}$ for a large $Q^{2}$ range between 0.1 and $1(\mathrm{GeV} / c)^{2}$. This measurement was done by detecting the recoil protons at a fixed angle between $68^{\circ}$ and $78^{\circ}$ from the elastic electron-proton scattering. A second measurement is underway at backward electron scattering angle of about $110^{\circ}$ for the hydrogen and deuterium targets for different incident beam energies of 362 and $687 \mathrm{MeV}$.

The axial form factor $G_{A}^{e N}$ has been considered for a long time as a quantity to be predicted for the extraction of $G_{E}^{s}$ and $G_{M}^{s}$ (see relation (4)). But, despite its unknown $Q^{2}$ behavior which implies a needed measurement for strange contribution at high $Q^{2}$, it contains information with theoretical and experimental implication. It can be expressed through the relation

$$
G_{A}^{e N}\left(Q^{2}\right)=G_{A}^{Z, N}\left(Q^{2}\right)+\eta F_{A}\left(Q^{2}\right)+R_{e} .
$$

The first term contains the charged current contribution (accessible in neutrino scattering) and the strange contribution to the axial for factor which was measured at $Q^{2}=0$ in deep inelastic scattering. The second term is the anapole form factor which corresponds to multiquark radiative correction and the last term is a one-quark radiative correction. Except for the charged current contribution, the $Q^{2}$-dependence of all these terms is unknown. The last two terms have been calculated theoretically at $Q^{2}=0$ but with large uncertainty.

The first experimental information on $G_{A}^{e N}$ has been obtained from the SAMPLE experiment at $Q^{2}=$ $0.1 \mathrm{GeV}^{2}$, where the extracted isovector nucleon axial form factor was found to be consistent with the theoretical estimate of Zhu [6]. 


\section{Experimental set-up in the forward-angle configuration}

The G0 experiment [7] has been performed in Hall C at Jefferson Laboratory. It requires high statistics (about $10^{13}$ elastic events) in order to measure very small asymmetries between 3 to $50 \mathrm{ppm}$ with a relative error close to $5 \%$. This requires beams with high intensity and high polarization. Also small helicity-correlated effects from the beam (intensity, position, ...) must be suppressed and if possible be uncorrelated to the detector and electronics response. The design of the experimental set-up has to achieve the highest possible data taking rates with a long cryogenic target and large acceptance of the detectors.

In the following we describe in more details all the items associated to the G0 set-up from the beam properties to the magnet, target, detectors and electronics.

\subsection{Electron beam}

The polarized electrons are provided by the interaction of a circularly polarized laser on the strained GaAs photocathode, which allows to select a particular transition of the crystal. This method allows to achieve high polarization $(\geq 70 \%)$, which will be reversed at $30 \mathrm{~Hz}$ frequency (defining a macro-pulse period (MPS) of $33 \mathrm{~ms}$ ) in less than $200 \mu \mathrm{s}$, by changing the polarity on a Pockels cell. In order to avoid time drift effects and to minimize the correlation of the helicity signal with the counting recording, the helicity will be randomly sequenced by quartet and will also be sent to the acquisition after a few MPS.

As previously mentioned, G0 requires high intensity $(40 \mu \mathrm{A})$ at a different mode than CEBAF is usually operating $(31 \mathrm{MHz}$ instead of $500 \mathrm{MHz})$. This mode uses a charge per bunch 16 times larger than the normal operating, which implies new requirements on the beam optics, due to space charge effects. The beam polarization, will be measured with an accuracy of about $2 \%$ using the standard Møller polarimeter available in Hall C.

An important issue in this kind of experiment is related to the systematic errors associated with the beam properties. The resulting false asymmetries, induced by possible correlations between the counting rates in the detectors and the beam parameters, as the beam charge, positions, angles and energy, have to be corrected with a good precision.

The way to minimize these systematic errors to an acceptable level is to keep these differences as low as possible so the run-averaged correction on the asymmetry remains lower than the statistical error $\left(\simeq 10^{-7}\right)$. Secondly, the beam parameters differences have to be measured accurately in such a way that the error on the correction (during the overall experiment) is kept as low as $5 \% \Delta A_{\text {stat }}$ $\left(\simeq 5 \cdot 10^{-9}\right)$. These beam requirements, which are summarized from each beam parameter on table 1, were all met during the G0 experiment.
Table 1. Challenging beam requirements and realizations for the G0 functioning. These are the differences between the two helicity states summed over the entire experiment.

\begin{tabular}{|lcc|}
\hline $\begin{array}{l}\text { Beam } \\
\text { parameters }\end{array}$ & $\begin{array}{c}\text { Differences } \\
\text { specifications }\end{array}$ & $\begin{array}{c}\text { Differences } \\
\text { measured }\end{array}$ \\
\hline CW current & $\leq 1 \mathrm{ppm}$ & $-0.14 \pm 0.32 \mathrm{ppm}$ \\
Position & $\leq 20 \mathrm{~nm}$ & $3 \pm 4 \mathrm{~nm}$ \\
Angle & $\leq 2 \mathrm{nrd}$ & $1 \pm 1 \mathrm{nrd}$ \\
Energy & $\leq 75 \mathrm{eV}$ & $24 \pm 4$ \\
\hline
\end{tabular}

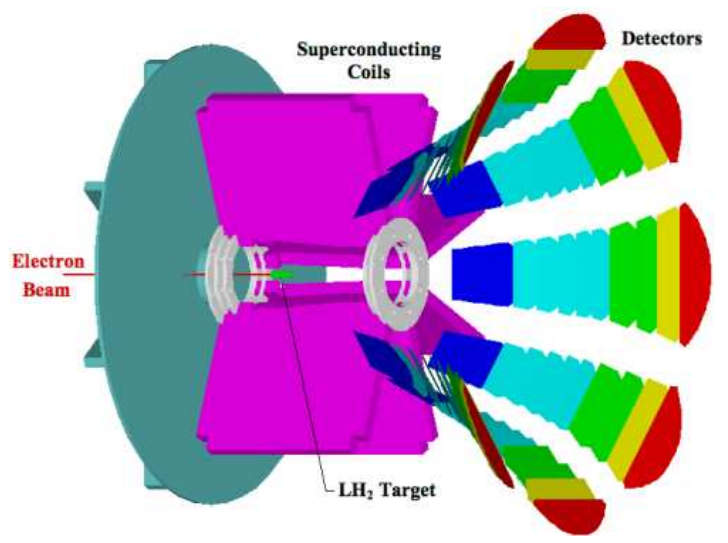

Fig. 1. G0 spectrometer.

\subsection{Target and spectrometer}

The G0 experiment will use a $20 \mathrm{~cm}$ target. Its design, which is based on the one used in the SAMPLE experiment, is optimized to reduce the energy loss on the scattered particles, and to minimize density variations up to $250 \mathrm{~W}$ of beam power deposited in the target. Also the target can be removed from the beam trajectory for diagnostic purposes and can be warmed independently of the spectrometer. It is fronted by a helium cell which is used to extend the entrance of the hydrogen cell and to eliminate variations in the effective $\mathrm{LH}_{2}$ thickness with beam position.

The spectrometer is the central piece of the G0 apparatus. It consists of a superconducting toroidal magnet with eight sectors placed around the beam axis (see fig. 1). The charged particles coming from the elastic scattering, produced in the target are selected in momentum by the magnetic field so that their location on the focal plane is independent of the interaction point along the beam axis. It has been designed to achieve large acceptance in solid angle which corresponds to a large- $Q^{2}$ acceptance from 0.1 to $1(\mathrm{GeV} / c)^{2}$.

The high magnetic field produces a bend angle of $35^{\circ}$ which is associated to a collimator geometry allowing to remove the direct view from the target and thus reduce the background coming from neutral particles in the detectors. Horizontal collimators are installed in order to define accurately the azimuthal acceptance, which is about $22^{\circ}$ for each octant, and prevent particles to be deviated in the neighboring octants. Also vertical collimators are used to 


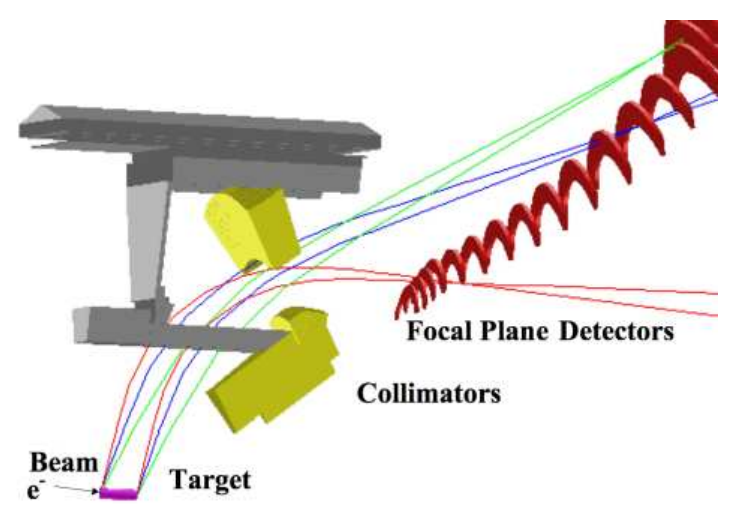

Fig. 2. Trajectories of protons elastically scattered for three different $Q^{2}=0.13,0.25$ and $0.7(\mathrm{GeV} / c)^{2}$.

restrict the $\theta$ angular acceptance to the one corresponding to the elastic scattering and thus reduce counting rates from inelastic protons and pions.

\subsection{The focal-plane detectors}

Detectors are located at the focal plane of the spectrometer for each of the eight octants defined by the coils. An octant is composed of 16 pairs of plastic scintillators coupled to light guides. The arc shape of each scintillator has been calculated from simulated trajectories of elastic protons created along the target at fixed transfers momentum $Q^{2}$. As shown in fig. 2, protons related to different $Q^{2}$ are scattered at a different location in the focal plane of the spectrometer and, for a fixed $Q^{2}$, all protons coming from different part of the target will be focused in the same detector.

The detector size have been optimized to limit the counting rates to $1-2 \mathrm{MHz}$. Also each detector is composed of a pair of scintillators separated by an aluminium or plastic plate. The coincidence between these two active layers allows to reduce significantly the background related to neutral particles like neutrons and $\gamma$ 's produced in the target and other materials. Because PMTs (Photomultiplier tubes) must be located far away from the spectrometer in relatively low magnetic-field regions, scintillators have been connected at each extremity to long light guides whose lengths range from $40 \mathrm{~cm}$ up to $2 \mathrm{~m}$. Their design has been carefully studied in order to optimize the light collection at the PMT.

The elastic protons are discriminated from other particles (protons, pions, etc.) using time of flight measurement (see fig. 4). In order to optimize the experimental time resolution, the detectors are placed transverse to the particle trajectory in order to reduce spread on the time of flight. Also the choice of BC408 Bicron as scintillating material has been made to insure a good timing resolution (less than $0.5 \mathrm{~ns}$ ) required for time measurement.

The time-of-flight spectrum associated with detector 15 is different compared to the other detectors because it is located at the maximum kinematic angle allowed for elastic protons. Thus the range in $Q^{2}$ is large, from 0.5

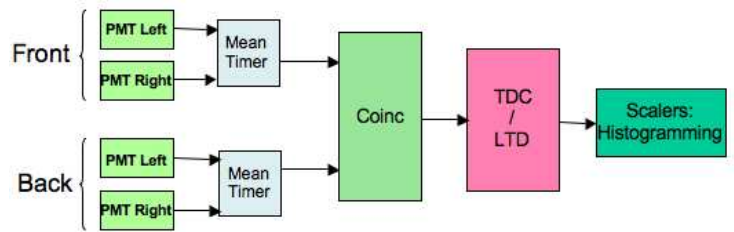

Fig. 3. Schematic design of the forward-angle electronics.

to $1(\mathrm{GeV} / c)^{2}$ and this is reflected in a wider time distribution associated to the elastic peak. Three different binnings in the time spectra were used to define different $Q^{2}$ ranges.

One has to notice that the French and North-American parts of the collaboration have provided each four octants which differ only in details concerning light guides and mechanical support.

\subsection{Electronics}

For the forward-angle measurement, the electronics needed for the asymmetry measurement has to provide time-of-flight information associated to each detector with rates ranging between 1 to $2 \mathrm{MHz}$. Also the electronics response has to be very stable whatever the experimental conditions are, to avoid any helicity-correlated systematic effects. For example the electronics deadtime has to be minimized and known with a good accuracy.

Two different electronics systems have been built by the North-American and French parts of the collaboration and each was used for half of the detectors. They allowed to study, in detail, different responses and related systematic effects. These two systems are based on the same concept as shown in fig. 3. The four analog signals, corresponding to the four PMTs of one detector, are sent into Constant Fraction Discriminators (CFD). The time information associated to left and right PMTs are meantime to cancel the time dependence versus the location of the interaction along the scintillator. Then the coincidence between the front and the back detectors is required to record the time-of-flight information. While the time range of $32 \mathrm{~ns}$ is fixed by the interval between two beam pulses, the time binning differs for the two electronics with $1 \mathrm{~ns}$ for the North-American and 250 ps for the French one. Then, the time information is histogrammed during one MPS and read out during the helicity reversal.

Finally, the charge and time distributions associated to each discriminator and meantimer will also be prescaled from a standard event-by-event Fastbus acquisition and will be used to monitor possible drifts of gains and time resolution of PMTs. Fastbus acquisition is also used for recording information related to the beam.

\section{G0 forward-angle results}

\subsection{From raw to physics asymmetries}

The data collected in 701 hours correspond to 101 coulombs of high-quality beams suited for parity violation 
Table 2. Asymmetries correction applied to raw asymmetries.

\begin{tabular}{|ll|}
\hline Source & Correction and uncertainty \\
\hline Residual deadtime & $\Delta A=0.05 \pm 0.05 \mathrm{ppm}$ \\
Residual helicity-correlated & $\Delta A=0.02 \pm 0.01 \mathrm{ppm}$ \\
beam properties & $\Delta A=0.71 \pm 0.14 \mathrm{ppm}$ \\
Beam leakage & $P e=73.7 \pm 1 \%$ \\
Beam polarization & $\Delta A=-1$ to $42 \pm 0.2$ to $9 \mathrm{ppm}$ \\
Background & $\left(\right.$ depending on $\left.Q^{2}\right)$ \\
\hline
\end{tabular}

experiments. Systematic studies show that the response of the spectrometer to beam changes is well understood. The distribution of the measured asymmetries calculated for each four helicity sequence is compatible with a Gaussian over five order of magnitude, and the measured width agrees at a level of few percent with the expected ones. Other searches for experimental asymmetries are through the polarization reversal using mechanical insertion of a $\lambda / 2$ wave plate. The asymmetries integrated over detectors change sign with no offset observed at the $0.15 \mathrm{ppm}$ level. Correction to beam changes, as well as the deadtime, are corrected within the analysis for each helicity state. Several sources of remaining non-physical asymmetries has been identified and corrected with very small absolute errors (see table 2). The beam leakage comes from the beam of other experimental Halls (A and B) into the Hall C. The beam leakage current is very small $(40 \mathrm{n} A / 40 \mu A)$ but the asymmetry is large (around $340 \mathrm{ppm}$ ). This induce a false asymmetry depending on the time of flight (TOF) and the beam intensity. The correction to be applied is measured using a region in TOF which is signal free. From the raw asymmetries measured, the various corrections are applied to give the physics asymmetries. The $Q^{2}$ of the measurement is then determined by simulation with $1 \%$ error and allow to interpolate the electromagnetic form factors to calculate the non-strange vector asymmetry $\left(A_{N V S}\right)$. The difference between $A_{\text {phys }}$ and $A_{N V S}$ gives the linear combination $G_{E}^{s}+\eta G_{M}^{s}$.

\subsection{Background overview}

The background in the experiment has been intensively studied using several targets to understand and measure the electro and photo production coming form the windows of the target and from the $\mathrm{LH}_{2}$ itself. The main contribution to the asymmetries is due to protons coming from hyperon weak-decay scattering inside the spectrometer. The simulation provides a very good understanding of these contributions but cannot be used for correction. These events are rare with very large positive asymmetries.

The background asymmetries are determined in two steps. First the background's yield is fitted over a large time spectra range, using a polynomial function (see fig. 4 , top panel). This gives the dilution factor $f(t)=\frac{Y_{\text {back }}(t)}{Y_{\text {meas }}(t)}$. Then, the background and elastic asymmetries are fitted

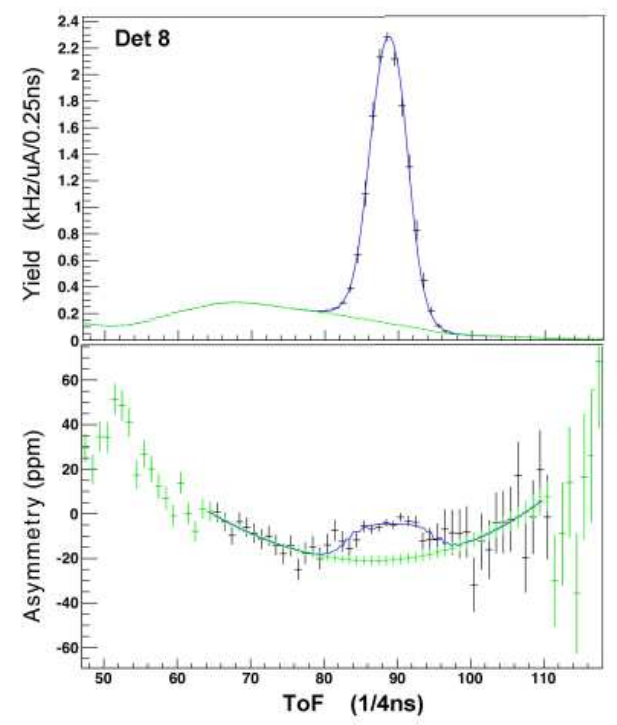

Fig. 4. Fit of the yield (top) and asymmetry (bottom) for detector 8 .

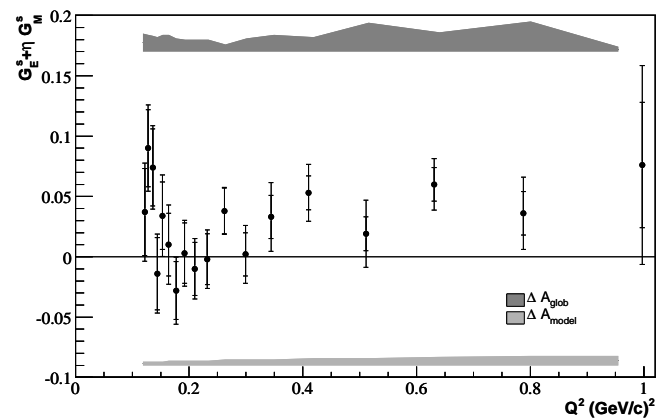

Fig. 5. Combination of electric and magnetic form factors obtained from the G0 forward-angle experiment.

(see fig. 4, bottom panel) according to

$$
A_{\text {meas }}(t)=(1-f(t)) A_{e l}+f(t) A_{b a c k}(t) .
$$

The elastic asymmetry is taken as constant, while the background one is taken as a polynomial. Conservative uncertainties are obtained by varying these quantities over a reasonable range. Due to the wide $Q^{2}$ range of the detector 15 (see sect. 3.3), three differents asymmetries are fitted for three differents $Q^{2}$ values $\left(0.51,0.63,0.79(\mathrm{GeV} / c)^{2}\right)$.

\subsection{Results}

The results of the experiment are shown in fig. 5. The linear combination $G_{s}^{E}+\eta G_{s}^{M}$ is the difference of the experimental asymmetry and the "no-vector-strange" asymmetry calculated from eq. (4) using $G_{s}^{E}=G_{s}^{M}=0$ for all values of $Q^{2}$. The error bars include the statistical uncertainty (inner) and the statistical plus point-to-point systematic uncertainties added in quadrature. The error bands correspond to the global systematic uncertainties 
from the experiment (upper), and from the quantities entering in $A_{N V S}$ (lower) (see [8] for details). This results show a non-trivial $Q^{2}$-dependence which may indicate two contributions of opposite sign. The G0 data are in agreement with other experiments for $Q^{2}$ values where data exist $\left(0.1,0.23\right.$ and $\left.0.48(\mathrm{GeV} / c)^{2}\right)$. Actually, independent extraction of $G_{s}^{E}$ and $G_{s}^{M}$ is only possible at $Q^{2}=0.1$. The last HAPPEX results [9] confirms a null $G_{s}^{E}$ with a better precision and a positive $G_{s}^{M}$. New measurements are underway with the G0 apparatus in the backward angle mode [10]. New data will soon be available for $Q^{2}=0.23$ and $0.6(\mathrm{GeV} / c)^{2}$, for hydrogen and deuterium target. This will allow the separation of the two terms (electric and magnetic) over a large $Q^{2}$ domain in order to get the $Q^{2}$-dependence of the strange quark contribution.

I would like to thank the organisers of PAVI 2006 for their great hospitality. I wish a successful running G0 data taking.
The G0 experiment is supported by DOE (U.S.), CNRS/IN2P3 (France), NSERC (Canada) and NSF (U.S.).

\section{References}

1. D.H. Beck, B. Holstein, Int. J. Mod. Phys. E 10, 1 (2001).

2. R. Tieulent, PhD Thesis, Grenoble University, ISN 02-27 (2002).

3. K.A. Aniol et al., Phys. Rev. Lett. 96, 022003 (2006); K.A. Aniol et al., Phys. Lett. B 635, 275 (2006).

4. PV A4 Collaboration (F.E. Maas et al.), Phys. Rev. Lett. 93, 022002 (2004); 94, 1520001 (2005).

5. SAMPLE Collaboration (D.T. Spayde et al.), Phys. Lett. B 583, 79 (2004).

6. S. Zhu et al., Phys. Rev. D 62, 033008 (2000).

7. R. Carr et al., in preparation.

8. D. Armstrong et al., Phys. Rev. Lett. 95, 092001 (2005).

9. D. Armstrong, this conference.

10. S. Kox, this conference. 